\title{
Determining Phonon Mean Free Paths from Observations of Quasiballistic Thermal Transport
}

\author{
A. J. Minnich* \\ Division of Engineering and Applied Science, \\ California Institute of Technology, Pasadena, California 91125, USA
}

(Received 6 August 2012; published 13 November 2012)

\begin{abstract}
The mean free paths (MFPs) of thermal phonons are mostly unknown in many solids. Recent work indicates that MFPs may be measured using experimental observations of quasiballistic thermal transport, but the precise relationship between the measurements and the MFP distribution remains unclear. Here, we present a method that can accurately reconstruct the MFP distribution from quasiballistic thermal measurements without any assumptions regarding the phonon scattering mechanisms. Our result will enable a substantially improved understanding of thermal transport in many solids, particularly thermoelectrics.
\end{abstract}

DOI: 10.1103/PhysRevLett.109.205901

PACS numbers: $65.40 .-\mathrm{b}$

Thermal transport at length scales comparable to phonon wavelengths and mean free paths (MFPs) is presently a topic of considerable interest [1-4]. Size effects caused by the macroscopic dimensions of a bulk sample were first considered by Casimir [5]. Recently, nanostructured materials such as nanowires, superlattices, and nanocomposites with strongly reduced thermal conductivities due to phonon scattering at interfaces and boundaries have been reported and are being assessed for use in thermoelectrics applications [6]. Phonon transport near nanoscale objects such as transistors is also of critical importance for heat dissipation in microelectronic devices [7].

Despite the crucial importance of the knowledge of phonon MFPs towards understanding and engineering these size effects, MFPs are largely unknown even for basic semiconductors. Traditionally, semiempirical expressions have been the only means to estimate MFPs [8]. Various experimental techniques such as photoacoustic wave propagation [9], inelastic neutron scattering [10], heat pulse techniques [11], a time-resolved x-ray diffraction technique [12], and others have some limitation such as a restriction on the sample type, accessible phonon frequency range, or applicable temperatures.

In recent years progress has been made on this difficult problem. First-principles calculations based on density functional theory have enabled the direct computation of MFPs in materials with simple crystal structures [13]. Experimentally, a recently introduced thermal conductivity spectroscopy technique allows MFPs to be measured over a wide range of length scales and materials using observations of quasiballistic thermal transport [14]. The technique is based on the fact that the heat flux of phonons with MFPs longer than a thermal length scale, such as the size of a heater, is strongly suppressed compared to the Fourier law prediction [15]. The MFP distribution is obtained by measuring the change in the effective thermal conductivity as the thermal length is systematically varied. In recent studies, the difference between the measured and actual thermal conductivity was assumed to be the contribution by those modes with MFPs longer than the thermal length [16]. MFPs have now been measured in silicon using this technique $[14,17]$.

Despite this advance, the relationship between the observed thermal conductivities, the imposed thermal length scale, and the MFPs in the material remains unclear. Thus far, the appropriate thermal length used to interpret the measurements has been estimated empirically by comparing the measurements to known MFP distributions provided by first-principles calculations $[14,17]$. For the many materials for which MFPs are completely unknown, including most thermoelectric materials, this empirical estimation will not suffice, and a more rigorous method to obtain the phonon MFPs is needed.

Here, we present a method that can accurately reconstruct the MFP distribution from quasiballistic thermal measurements without any assumptions regarding the phonon scattering mechanisms. The method is based on the solution of a common inverse problem with the experimental measurements as input. This result paves the way for accurate measurements of MFPs and is expected to lead to a substantially improved understanding of thermal phonon scattering in numerous classes of solids of scientific and technological interest, such as semiconductors, polycrystals, and disordered materials.

We begin by analyzing the thermal conductivity spectroscopy technique. Mathematically, the experiment provides $M$ measurements of the thermal conductivity $k_{i}$ as a function of a thermal length scale $L_{i}$. As $L_{i}$ decreases, $k_{i}$ decreases by an amount corresponding to the contribution of phonon modes with MFP comparable to the thermal length. For a particular heating geometry, a heat flux suppression function $S\left(\Lambda_{\omega} / L_{i}\right)$ describes how the modes are suppressed as a function of the thermal length $L_{i}$ and the MFPs $\Lambda_{\omega}$. For example, for the 1D transient grating geometry, the function has been shown to be [18]

$$
S\left(q \Lambda_{\omega}\right)=\frac{3}{q^{2} \Lambda_{\omega}^{2}}\left(1-\frac{\tan ^{-1}\left(q \Lambda_{\omega}\right)}{q \Lambda_{\omega}}\right),
$$


where in this case $q=2 \pi / \lambda$ and $\lambda$ is the grating wavelength.

The measurements $k_{i}$ and the suppression function $S$ are related to the differential and cumulative MFP distributions $f\left(\Lambda_{\omega}\right)$ and $F\left(\Lambda_{\omega}\right)$, respectively, by

$$
k_{i}=\int_{0}^{\infty} S\left(x_{i}\right) f\left(\Lambda_{\omega}\right) d \Lambda_{\omega}=\int_{0}^{\infty} q_{i} K\left(x_{i}\right) F\left(\Lambda_{\omega}\right) d \Lambda_{\omega},
$$

where $x=q \Lambda_{\omega}, \quad F\left(\Lambda_{\omega}\right)=\int_{0}^{\Lambda_{\omega}} f\left(\Lambda^{\prime}\right) d \Lambda^{\prime}$, and $K(x)=$ $-d S / d x$. Here we have used integration by parts and the fact that $F(0)=0$ and $K(\infty)=0$ to eliminate the constant. We will focus on determining the cumulative distribution $F\left(\Lambda_{\omega}\right)$ and refer to it as the MFP distribution.

Equation (2) is an inhomogeneous Fredholm integral equation of the first kind that occurs in many fields of science and engineering as an inverse problem. For example, for certain forms of the kernel $K$, solving Eq. (2) represents a deconvolution.

The desired MFP distribution is thus given by the solution to the ill-posed problem of Eq. (2). To allow the equation to be numerically solved we discretize the integral using Gaussian quadrature, yielding a linear system of equations for the unknown MFP distribution $F_{j}$,

$$
\sum_{j=1}^{N} A_{i, j} F_{j}=k_{i}
$$

where $N$ is the number of integration points, $F_{j}=F\left(\Lambda_{j}\right)$ is the desired MFP distribution, $A_{i, j}=K\left(q_{i} \Lambda_{j}\right) q_{i} \beta_{j}$ has dimension $M \times N$, and $\Lambda_{j}$ and $\beta_{j}$ are the quadrature points and weights, respectively.

The matrix $A$ is singular even for $M=N$ and cannot be solved by inversion. This degeneracy is expected because the averaging caused by the kernel results in a permanent loss of information about the solution, resulting in a singular linear system.

Equation (3) would be impossible to solve if no other information were known about $F$. However, because $F$ is a cumulative distribution function (CDF), it is subject to the following restrictions: (i) $F(0)=0$, (ii) $F(\infty)=1$, and (iii) $F$ is non-negative and monotonically increasing. Furthermore, the MFP distribution is unlikely to have abrupt steps because it is spread over such a wide range of MFPs. $F$ must thus obey some type of smoothness restriction.

We can incorporate these requirements into the solution of Eq. (3) by reformulating the problem as a convex optimization. We seek to minimize a penalty function $P$ subject to the 3 conditions above, where $P$ is defined as

$$
P=\|A F-k\|_{2}^{2}+\eta\left\|\Delta^{2} F\right\|_{2}^{2} .
$$

Here, $\Delta^{2} F=F_{j+1}-2 F_{j}+F_{j-1}$ is the second difference operator and $\|\cdot\|_{2}$ is the 2-norm. The first term on the right-hand side of Eq. (4) enforces that the solution satisfies Eq. (3), while the second term enforces smoothness in the solution. $\eta$ controls the relative weight of the smoothness penalty. We find that the solution is not sensitive to the particular value of $\eta$ within a certain range, and values from 0.5 to 1.5 yield similar results. If $\eta$ is too small, the solution will have unphysical jumps, while if $\eta$ is too large the solution will not satisfy the linear system. We use $\eta=1$ for all the results in this work.

One benefit of using convex optimization is that all the knowledge of the MFP distribution can be used in the calculation. For example, measurements can be taken using different experimental techniques and incorporated into Eq. (3) by adding a row to the matrix and using the appropriate kernel. If an asymptotic form of the MFP distribution is known, this requirement can also be specified.

We can now formulate a general procedure for measuring the MFP distribution using observations of quasiballistic thermal transport. First, measure the effective thermal conductivity $k_{i}$ over several thermal lengths. Next, obtain the kernel $K$ for a specified heater geometry by solving the Boltzmann transport equation (BTE). Finally, use convex optimization to solve Eq. (3) for the MFP distribution.

To illustrate this procedure, we perform a numerical "experiment" in which we use the BTE to obtain both the kernel and the effective thermal conductivities. We first briefly discuss our solution of the BTE. The equation is given by [19]

$$
\frac{\partial e_{\omega}}{\partial t}+\mathbf{v} \cdot \nabla_{\mathbf{r}} e_{\omega}=-\frac{e_{\omega}-e_{\omega}^{0}}{\tau_{\omega}},
$$

where $e_{\omega}$ is the desired distribution function, $\omega$ is the angular frequency, $e_{\omega}^{0}$ is the equilibrium distribution function, $\mathbf{v}$ is the group velocity, and $\tau_{\omega}$ is the frequency dependent relaxation time.

We solve this equation using a recently introduced deviational Monte Carlo (MC) method [20]. This technique solves the BTE by simulating the advection and scattering of computational particles denoted phonon bundles, which represent some number of actual phonons. To implement the simulation, a computational domain is discretized into cells, and the phonon dispersion is divided into approximately 1000 frequency bins. The domain is initialized with phonon bundles corresponding to the initial temperature distribution. Because all the boundaries are adiabatic, the simulation runs with a fixed number of phonon bundles. At each time step, the bundles are advected, indexed into cells to sample their temperature, and scattered. The calculation proceeds in this manner until the desired simulation time.

We perform our simulations for crystalline silicon, using the experimental dispersion in the [100] direction and assuming the crystals are isotropic. The numerical details concerning the dispersion and relaxation times are the same as those described in our recent work [21]. Only phonon-phonon scattering is considered for simplicity. 
The code was validated by performing the same two test problems discussed by Peraud et al. [20]; excellent agreement is observed in both cases.

The initial deviational temperature profile corresponds to that for a 1D transient grating geometry, $\Delta T(z, t=0)=$ $\cos q z$, where $z$ is the spatial coordinate. The computational domain length $L_{z}=\lambda / 2$. All of the boundaries are adiabatic by symmetry and are enforced as specular reflection.

We begin the MFP measurement procedure by obtaining the effective thermal conductivities. As would occur in an experiment, these values are determined by fitting the transient temperature decays for each grating wavelength to the Fourier law solutions. Example decay curves for two grating wavelengths, along with the Fourier law solution $\Delta T_{f}(z=0, t)=\exp \left(-\alpha_{z} q^{2} t\right)$, are presented in Fig. 1(a). For these grating wavelengths in the micron range, the BTE predicts a slower thermal decay, and therefore thermal conductivity, than does the heat equation, as expected. We obtain effective thermal conductivities $k_{i}$ for each decay curve by determining the exponential decay constant and using the expression for $T_{f}$, thereby providing the right-hand side of Eq. (3).

Next, we need to determine the kernel $K$, which is related to the heat flux suppression function $S$ for the heater geometry by $K=-d S / d x$, where $S=Q_{m} / Q_{f}$ is defined as the ratio of the observed heat flux $Q_{m}$ to the Fourier heat flux $Q_{f}$. The Fourier law heat flux $Q_{f}=C_{\omega} v_{\omega} \Lambda_{\omega} / 3$, where $C_{\omega}$ and $v_{\omega}$ are the specific heat per frequency and the group velocity, respectively. The $\mathrm{MC}$ heat flux $Q_{m}$ is obtained by summing the heat flux contributions for each phonon mode over the duration of the simulation, giving a time-averaged heat flux for each mode, and will be smaller than $Q_{f}$ for phonons with long MFPs due to quasiballistic effects.

Figure 1(b) shows the Fourier and MC spectral heat flux, or the heat flux per unit phonon frequency, plotted as a function of the MFP. The suppression function $S$ given by
Eq. (1) is also shown and is in excellent agreement with the numerical results. The resulting kernel has the shape of a broadened delta function. While in this case an analytical expression exists for the kernel, our MC calculation is capable of determining the appropriate kernel for any geometry by fitting $S$ with splines and taking a numerical derivative.

We emphasize that in obtaining $K$, we have not made any assumption regarding the MFPs in the material to be studied. This procedure only determines the suppression of phonons of different MFPs for a given heater geometry and is independent of the MFPs used in the calculation.

The last step is to use convex optimization to solve for the MFP distribution. For this step we use CVX, a package for specifying and solving convex programs $[22,23]$. The linear system of equations in Eq. (3) is obtained by using Gaussian quadrature with $N=50$ to discretize the integral in Eq. (2). We use $M=12$ numerically calculated "measurements" of the effective thermal conductivity. For this small optimization problem the MFP distribution $F_{j}$ is obtained in seconds.

The analytic MFP distribution, the numerical data with added noise to simulate experimental uncertainty, and the MFP distribution predicted by the convex optimization are shown in Fig. 2. The reconstructed distribution is in excellent agreement with the analytic distribution over nearly the full range of MFPs even though the data consist of only 12 data points for grating wavelengths between 1-15 microns. We purposely only included this small number of data points so that the data correspond to experimentally accessible grating wavelengths, but using measurements spanning a wider range of lengths increases the accuracy of the reconstruction. If all the simulated data points are used the full MFP distribution can be reconstructed essentially exactly (not shown).

That the MFP distribution can be accurately determined even outside of the length range of the measurements may seem surprising given that the problem is underdetermined.
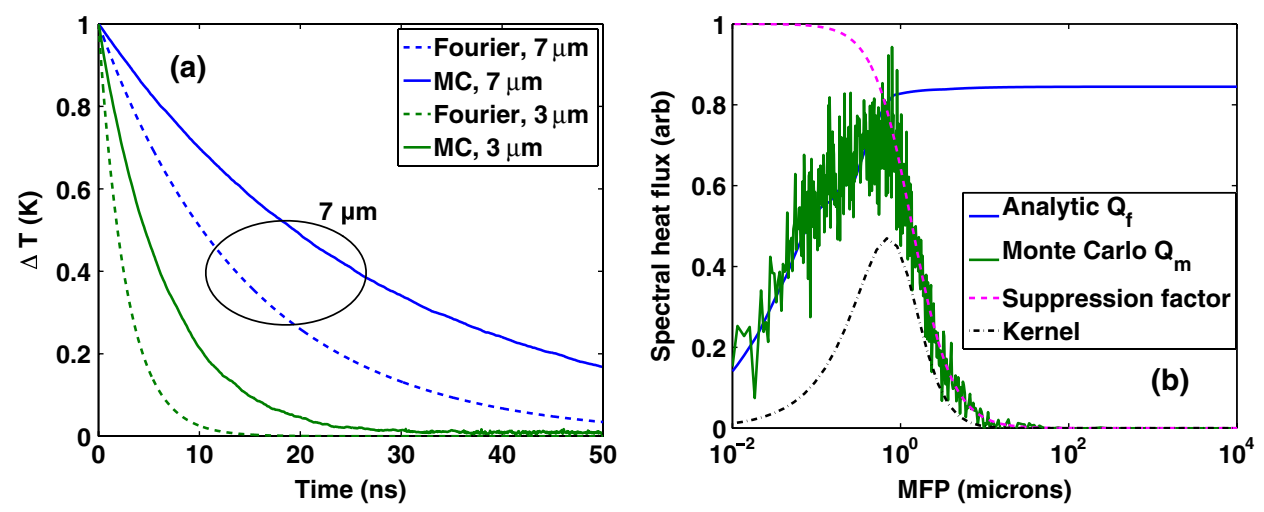

FIG. 1 (color online). (a) Deviational temperature decay curves $\Delta T(z=0, t)$ predicted by the numerical Monte Carlo solution (solid lines) and the heat equation (dashed lines) for the two transient grating wavelengths indicated. (b) Spectral heat flux (per phonon frequency) plotted as a function of MFP $\Lambda_{\omega}$ for $\lambda=7 \mu \mathrm{m}$. The Fourier heat flux $Q_{f}$ (solid line), the MC heat flux $Q_{m}$ (rapidly varying line), the suppression function $S\left(q \Lambda_{\omega}\right)$ (dashed line), and the kernel $K\left(q \Lambda_{\omega}\right)$ (dash-dotted line) are shown. 


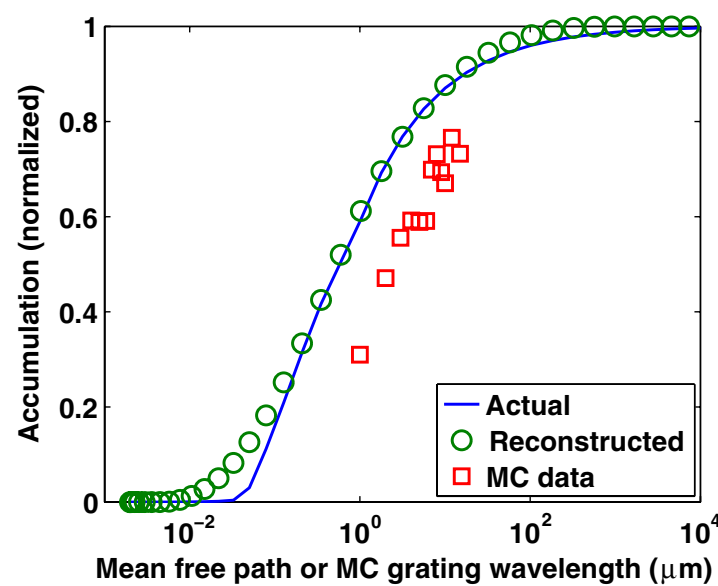

FIG. 2 (color online). Actual MFP distribution for silicon (line), the reconstructed MFP distribution (circles), and the effective thermal conductivities obtained from the MC simulations with artificially added noise (squares). The $x$ axis corresponds to the MFP for the two distributions (line and circles) and to the MC grating wavelength for the thermal conductivity data (squares). The reconstructed distribution agrees with the actual distribution to within 5\% over nearly the full MFP range. The distribution can be reconstructed nearly exactly over the full MFP range if data spanning a wider range of length scales is used.

This reconstruction is possible is due to the wide breadth of the kernel $K$ and the previously described constraints on $F$. As shown in Fig. 1(b), $K$ spans 3 orders of magnitude of MFPs. While the averaging induced by this kernel results in the loss of some information about the solution, it also expands the range of MFPs involved in the reconstruction. This fact, combined with the restrictions on $F$ because it is a CDF, constrains the solution and allows the distribution to be reconstructed over a larger range than that of the measurements. However, MFPs for which the kernel $K \approx 0$, such as those between 10-100 nm in Fig. 2, cannot be uniquely determined because the linear system imposes no constraint on these modes.

As an application of our method, we examine recently reported thermal conductivity measurements of a $390 \mathrm{~nm}$ $\mathrm{Si}$ membrane at room temperature [17]. The measurements were performed using the transient grating method such that the heat transport was entirely in-plane, allowing the suppression function considered in this work to be used. Following the procedure as above, we obtain the result shown in Fig. 3. The measured MFP distribution is in good agreement with an estimate obtained using firstprinciples calculations of MFPs and accounting for diffuse scattering from the membrane sidewalls using a Sondheimer calculation $[17,24]$. We note that while the MFP distribution is accurately reconstructured to within $15 \%$ using the present method, sharp features such as the kink in the actual MFP distribution at around $\Lambda_{\omega}=$ $200 \mathrm{~nm}$ cannot be resolved due to the averaging induced by the kernel.

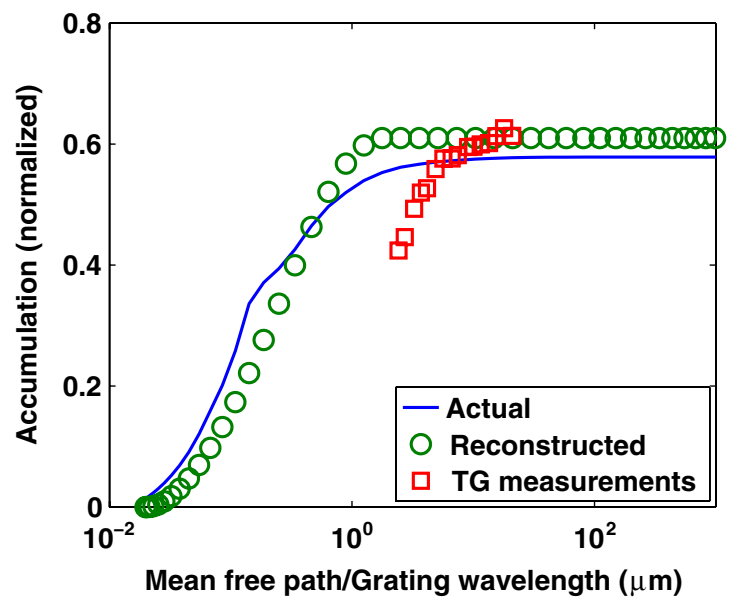

FIG. 3 (color online). The MFP distribution in a $390 \mathrm{~nm}$ thick $\mathrm{Si}$ membrane at room temperature (circles) reconstructed from recently reported transient grating measurements (squares) [17]. The $y$ axis is normalized to the bulk thermal conductivity value of Si. An analytic calculation (solid line), which was computed from a first-principles calculation of the MFP distribution in $\mathrm{Si}$ and accounting for diffuse scattering from the membrane boundaries, agrees with the reconstructed distribution to within approximately $15 \%$.

Our analysis indicates that heat is primarily carried by phonons with MFPs smaller than 1 micron in the membrane, with the majority of the heat being contributed by phonons with $\Lambda_{\omega}<500 \mathrm{~nm}$. We can thus conclude that most thermal phonons are completely diffusely scattered at the membrane boundaries because most of the heat is carried by phonons with MFPs shorter than the membrane thickness. This physical insight into surface roughness scattering would be difficult to obtain without knowledge of the MFPs provided by the method. Further study of variable thickness membranes at different temperatures would yield additional insight into the phonon frequency dependence of the surface roughness scattering mechanism.

In summary, we have presented a method that can reconstruct the phonon MFP distribution from observations of quasiballistic thermal transport without any assumptions regarding the phonon scattering mechanisms. We expect the method to play an important role in advancing our understanding of thermal transport in numerous solids of technological importance, particularly thermoelectric materials.

The author would like to thank J. A. Tropp for help with the convex optimization, J.P. Peraud and Q. Hao for help with the MC simulations, and A. A. Maznev for useful discussions. This work was sponsored in part by Robert Bosch LLC through Bosch Energy Research Network Grant No. 13.01.CC11. This work was also supported in part by a start-up fund from the California Institute of Technology. 
*aminnich@caltech.edu

[1] J.-K. Yu, S. Mitrovic, D. Tham, J. Varghese, and J. R. Heath, Nature Nanotech. 5, 718 (2010).

[2] A. I. Hochbaum, R. Chen, R. D. Delgado, W. Liang, E. C. Garnett, M. Najarian, A. Majumdar, and P. Yang, Nature (London) 451, 163 (2008).

[3] G. Pernot, M. Stoffel, I. Savic, F. Pezzoli, P. Chen, G. Savelli, A. Jacquot, J. Schumann, U. Denker, I. Mönch et al., Nature Mater. 9, 491 (2010).

[4] M. C. Wingert, Z. C. Y. Chen, E. Dechaumphai, J. Moon, J. Kim, J. Xiang, and R. Chen, Nano Lett. 11, 5507 (2011).

[5] H. Casimir, Physica (Amsterdam) 5, 495 (1938).

[6] R. J. Mehta, Y. Zhang, C. Karthik, B. Singh, R. W. Siegel, T. Borca-Tasciuc, and G. Ramanath, Nature Mater. 11, 233 (2012).

[7] E. Pop, Nano Res. 3, 147 (2010).

[8] C. Herring, Phys. Rev. 95, 954 (1954).

[9] C. Thomsen, H. T. Grahn, H. J. Maris, and J. Tauc, Phys. Rev. B 34, 4129 (1986).

[10] O. Delaire, J. Ma, K. Marty, A. F. May, M. A. McGuire, M. Du, D. J. Singh, A. Podlesnyak, G. Ehlers, M. D. Lumsden et al., Nature Mater. 10, 614 (2011).

[11] R. J. von Gutfeld and A. H. Nethercot, Phys. Rev. Lett. 12, 641 (1964).

[12] M. Highland, B. C. Gundrum, Y. K. Koh, R. S. Averback, D. G. Cahill, V. C. Elarde, J. J. Coleman, D. A. Walko, and E. C. Landahl, Phys. Rev. B 76, 075337 (2007).
[13] A. Ward and D. A. Broido, Phys. Rev. B 81, 085205 (2010).

[14] A. J. Minnich, J. A. Johnson, A. J. Schmidt, K. Esfarjani, M. S. Dresselhaus, K. A. Nelson, and G. Chen, Phys. Rev. Lett. 107, 095901 (2011).

[15] G. Chen, J. Heat Transfer 118, 539 (1996).

[16] Y. K. Koh and D. G. Cahill, Phys. Rev. B 76, 075207 (2007).

[17] J. A. Johnson, A. A. Maznev, J. K. Eliason, K. A. Nelson, A. J. Minnich, G. Chen, J. Cuffe, T. Kehoe, and C. M. S. Torres, arXiv:1204.4735.

[18] A. A. Maznev, J. A. Johnson, and K. A. Nelson, Phys. Rev. B 84, 195206 (2011).

[19] A. Majumdar, J. Heat Transfer 115, 7 (1993).

[20] J. M. Péraud and N. G. Hadjiconstantinou, Phys. Rev. B 84, 205331 (2011).

[21] A. J. Minnich, G. Chen, S. Mansoor, and B.S. Yilbas, Phys. Rev. B 84, 235207 (2011).

[22] M. Grant and S. Boyd, in Recent Advances in Learning and Control, edited by V. Blondel, S. Boyd, and $\mathrm{H}$. Kimura, Lecture Notes in Control and Information Sciences (Springer-Verlag, Berlin, 2008), p. 95, http:// stanford.edu/ boyd/graph_dcp.html.

[23] M. Grant and S. Boyd, CVX: Matlab software for disciplined convex programming, version $1.21, . . / . / \mathrm{cvx}$ (2011).

[24] A. S. Henry and G. Chen, J. Comput. Theor. Nanosci. 5, 141 (2008). 